\title{
Europäische Lektüren jenseits von Europa: Weltliteratur, literarischer Kanon und die Bildung in Europa
}

\section{JAVIER GÓMEZ-MONTERO}

\begin{abstract}
European Readings beyond Europe: World Literature, Literary Canon and Education in Europe. The present essay discusses the concepts "literary canon", "world literature", "classic authors", and "literary masterworks". Moreover, it summarises their origins and describes the usage of these concepts. The aim is to update these terms to current validity and to suggest an appropriate use of them in the educational and academic realm.

For this purpose, some major works of European literature are contrasted with modern bestseller books (e.g. "high literature" as for instance "Bildungsroman" with "amusement novels", fantasy, etc.). In addition to that, the article contrasts private with educational and academic readings (literary works read by students at universities and schools). Merging the conclusions together with the pertinent anthropological criteria, the essay suggests a programme of European readings that does not refuse to take into account the concepts of classic authors (Calvino) and of Bildung (Schwanitz). At the same time, it responds to the social reality of our time and of our European cities.

Overall, this approach mediates between Italo Calvino's justification of reading classic authors and Harold Bloom's idea of reading as a lonely, quiet, and profound exercise, at the same time emotional as well as existential, carried out with a certain dose of (self-)irony. On his part, Calvino refused aesthetic criteria, such as outstanding unity and exemplary harmony, to characterise a reading as an extreme experience and process of initiation at the interfaces where classic authors, canon, and 'Weltliteratur' connect with the reader's individual experience. In doing so, Calvino sticks to the individual practice of reading and provides 15 arguments in favour of books that are, on the one hand, sustainably present in the collective memory as well as in the individual subconscious and, on the other hand, contribute to individual and collective self-determination. They assign a fund of readings with permanent validity because of their constant renewal and enduring effect over the centuries, in combination with their impact on the individual biographies of single persons.

In this sense, the article argues in favour of establishing humanistic canons at universities and schools that can make use of the performative impulses a reference system like this offers, in order to contribute to more complex ideas than those texts that are produced by the consumer society and the mass media day by day. Getting to know oneself and the others, deepening the mutual understanding - this could be supported by a canon of European literature.
\end{abstract}

Keywords: literary canon, world literature, classic authors, literary masterworks, reading, humanistic canons at universities and schools

DOI: http://dx.doi.org/10.12697/IL.2013.18.2.01 
Das Projekt University and School for a European Literary Canon (ELiCa) ist ein europäisches Projekt mit dem Ziel, ein Korpus an literarischen Werken aus dem europäischen Raum zu profilieren, das den Unterricht in Schulen und Universitäten bereichern kann. ${ }^{1}$

Wer sich einer solchen Aufgabe stellt, sieht sich rasch mit so kontrovers diskutierten Begriffen wie ,Weltliteratur' und ,Kanon' konfrontiert. Der Erfolg beider Konzepte verdankt sich ihrer suggestiven Kraft und Komplexität, zugleich aber auch dem Unbehagen, das sie auslösen. Wie auch immer man zu ihnen steht, sie stellen eine Herausforderung dar. Zu Beginn soll daher versucht werden, ,Weltliteratur' und ,Kanon' in den Rahmen eines spezifischen Kontextes der Bildung in Europa - verstanden im Sinne der Schul- bzw. Hochschulausbildung, aber auch einer Allgemeinbildung der Europäer - zu stellen und ihre nicht nachlassende Aktualität im Zusammenhang mit gesellschaftlichen Praktiken und mit der Funktion von Literatur innerhalb der Bildungssysteme Europas zu betrachten, insbesondere im Gymnasialunterricht, ${ }^{2}$ aber auch an der Universität. Selbstverständlich gleicht jede Generation diese Konzepte an die jeweiligen politischen, intellektuellen und beruflichen Horizonte und Kontexte an, und insbesondere der Begriff, Weltliteratur' hat sich seit seiner Entstehung grundlegend gewandelt. Ich werde eingangs daran erinnern, was Goethe unter,Weltliteratur' verstand, dann umreißen, welche Bedeutungserweiterung ,Weltliteratur' gegenwärtig erfährt, und im Anschluss einen Vorschlag zur Reformulierung wagen. Zudem hat es sich im Rahmen unserer Untersuchung als ratsam erwiesen, die Diskussion um das Konzept eines literarischen Kanons und dessen Anwendung einzugrenzen auf einen strategischen Vorschlag: gemeint sind Texte mit sozialer Relevanz und Akzeptanz, deren Vermittlung in Unterricht und Studium als unerlässlich erachtet wird. Davon ausgehend werde ich ein eher pragmatisches Modell skizzieren, das die Kategorien ,Weltliteratur' und ,Kanon' zurückverwandelt in eine Praxis des Lesens und in Lektüreprogramme, die sich auf die europäische Literatur konzentrieren. Ich gehe dabei von der Prämisse aus, dass alle ernstzunehmenden Vorschläge zugunsten eines literarischen Kanons die Prinzipien der subjektiven Auswahl und der strategischen Perspektivierung anerkennen.

1 Der vorliegende Beitrag entstand im Kontext des Projekts. Siehe dazu auch: Baratta, Grossi, Pupolin 2012 und die Kieler homepage: www.uni-kiel.de/elica.

2 In Spanien entsprechen dem Schulen mit Bachillerato-Abschluss (bzw. bachillerato superior), in Italien liceo und lycée in Frankreich. 


\section{Weltliteratur bei Goethe und heute}

,Weltliteratur' ist ein Begriff, der auf den Aufklärer Christoph Martin Wieland zurückgeht und den Goethe in wenigen Briefen, Notizen und Gesprächen auf unsystematische Weise, aber nachhaltig in Umlauf brachte. Damit befand sich der Begriff in Gesellschaft zahlreicher weiterer Wortprägungen wie ,Weltgeist', ,Weltseele' oder, Weltfrömmigkeit', die um 1800 herum aufkamen, wieder verblassten oder auch ergänzt wurden, wie ,Weltkultur oder das von der UNESCO institutionalisierte ,Weltkulturerbe zeigen. Heute gebrauchen wir ,Weltliteratur' in nahezu überzeitlichem Sinne, aber Goethe wendete den Begriff in einem präzisen historischen Rahmen an - unter bestimmten politischen, wirtschaftlichen und technischen Voraussetzungen, die den Schriftstellern seiner Zeit neue Möglichkeiten der wechselseitigen Kommunikation, des literarischen Austauschs und der intensiveren Kenntnis ihrer Werke eröffnete (vgl. dazu Lamping 2010). Die Zeit um 1800 war zugleich die Epoche der Zeitschriften und Rezensionen, der Übersetzungen und Briefwechsel; sie erlaubte der literarischen Elite Europas - besonders in Deutschland, Frankreich, England und Italien -, ein Netz der intensiven Kommunikation aufzubauen, das die Überwindung der Grenzen zwischen den Nationalliteraturen einforderte und dessen Projektionsradius auch die spanische, polnische, nordamerikanische und sogar die chinesische oder arabische Literatur umfasste. Das Wissen vom jeweils Anderen und das angemessene Verständnis der Nationen untereinander, aber auch die Kompensation ihrer partikularistischen Interessen und ein Ausgleich für aufbrausende Nationalismen gehörten zum erklärten Programm der ,Weltliteratur' in Goethes Sinne, die so eine soziale und politische Funktion erfüllen konnte und sollte (vgl. Bohnenkamp 1999).

,Weltliteratur' meint bei Goethe also in erster Linie die Kontakte zwischen Autoren und Texten wie auch die Texte, in denen diese Kontaktpflege stattfindet, „die Bildung eines Ideenverkehrs zwischen den Nationen” (Birus 1995: 27). Schon wenig später tritt an die Stelle dieses empirischen Befundes eine normative Bedeutung, die sich ausschließlich auf die Texte bezieht und die zahlreichen geläufigen Kanon-Konzepten zugrunde liegt.

Heute hat die Globalisierung neue Formen der literarischen Kommunikation hervorgebracht: die dem Internet eigenen nicht-hierarchischen Auswertungs- und Konsummodelle (in denen die bloße Dynamik ästhetische Kriterien ersetzt), die neuen Vertriebstechniken literarischer Texte oder - allgemeiner - textueller Erzeugnisse, die Kommunikation und Verbreitung befördern, ohne nach der Produktqualität zu fragen, ${ }^{3}$ die Rolle des Internets als

3 Diese Entwicklung thematisiert Manfred Schmeling in seinem Beitrag „Ist Weltliteratur wünschenswert? Fortschritt und Stillstand im modernen Kulturbewußtsein” (Schmeling 1995). 
Ort überbordender Text(re)produktion und schließlich das Diktat des Marktes. Es liegt daher nahe, dass ,Weltliteratur' heute jenseits ihrer Konzeption als kanonische Summe von literarhistorisch relevanten Werken auch Folgendes bedeutet: Werke, die weltweit rezipiert werden, in denen sich die literarischen Diskurse intensivieren und in ihren jeweiligen Ausprägungen sichtbar werden.

In diesem Zusammenhang stellen sich Fragen: Wird die Zugehörigkeit eines Textes zur ,Weltliteratur Ansatz einiges mit Gerald Gillespies also zukünftig an seinem Verkaufserfolg gemessen? Wird der Konsum mithin zum ausschließlichen Richtwert für ihre Relevanz? Ist der bildungsrelevante Charakter ihrer Lektüre noch von Bedeutung? Wird Englisch als literarische, Weltsprache' die Sprache einer solchen,Weltliteratur' sein? Die im Rahmen des Projekts University and School for a European Literary Canon durchgeführte Umfrage mit spezifischen Fragebögen hat - zumindest für Deutschland - drei wesentliche Befunde ergeben: erstens die große Divergenz zwischen der privaten und der institutionalisierten Lektüre an weiterführenden Schulen - speziell in der Oberstufe des Gymnasiums - oder an der Universität, zweitens eine fehlende Differenzierung zwischen populären Genres (etwa Fantasy-Roman oder Thriller) und einer anspruchsvollen intellektuellen Literatur und drittens der deutliche Einfluss von Kino, Fernsehen und neuen Medien auf die Lektüre von Schülern, Studenten und Lesern im Allgemeinen. Dieses Ergebnis zeigt, dass ein für den Unterricht an Schule und Universität zu entwerfender europäischer Kanon ein Gegengewicht darstellen sollte und sich an anthropologisch-humanistischen Kriterien orientieren könnte, um dem Lesepensum im Unterricht eine performative Funktion im Einklang mit aktuellen gesellschaftlichen Veränderungen und den neuen technologischen Bedingungen einzuschreiben. Angesichts der gegenwärtigen Multi- und Transkulturalität sollten ferner spezifische Leistungen europäischer Literatur in Anschlag gebracht werden. Dazu gehören sowohl das kulturelle Selbstverständnis, das die europäischen Nationen in ihren Literaturen zum Ausdruck gebracht haben, als auch die symbolischen und moralischen Grundlagen ihrer Gesellschaften, die sich in den literarischen Texten abbilden. Ein weiterer Schritt wäre dann die pragmatische Verhandlung dieser Grundlagen durch individuelle und kollektive Subjekte, denn zu den herausragenden Leistungen moderner europäischer Literatur zählt die Diskussion ethischen Verhaltens ebenso wie die Darstellung des individuellen und kollektiven Unbewussten, der Albträume einer Gesellschaft, das Ausloten sich entziehender Seiten des Bewusstseins und die Abbildung extremen menschlichen Verhaltens in allen Schattierungen.

Ergebnis dieser Überlegungen sollte also ein durch die Bildungsinstitutionen gefördertes Programm sein, dessen Durchführung sich, wie gesagt, als Gegengewicht zu den erwähnten Prozessen versteht und das die gesell- 
schaftsrelevante Dimension der ,Weltliteratur' sichern soll. Denn Literatur, davon gehen wir aus, verändert das Bewusstsein und hat insofern eine positive Auswirkung auf die Entwicklung des Individuums und auf das gegenseitige Verständnis gesellschaftlicher Gruppen.

Immerhin zeigen unsere Erfahrungen mit Oberstufenschülern auch, dass Figuren wie Antigone, Phaedra, Madame Bovary, Don Quijote, Faust, Romeo und Julia oder Don Juan durchaus attraktiv sind. Die Vermittlung der Texte im Unterricht und die Annäherung an diese Figuren erfordern natürlich spezifische didaktische Strategien (vgl. insb. Sommerfeldt 2011: 151-174), zum Beispiel basierend auf der Identifikation, der aktiven Beteiligung (etwa mit Rollenspielen) sowie einer schriftlichen, mündlichen oder theatralischen Performanz, wobei sich das Kriterium des Fremdsprachenerwerbs zusätzlich positiv auswirkt.

Alle diese literarischen Figuren gehören zur, Weltliteratur', deren Kennzeichen Zeitlosigkeit und eine weltumspannende Rezeption sind und deren Vertreter mit den Klassikern im Sinne Italo Calvinos (Calvino 2003) übereinstimmen. Schon seit längerem hat sich der eurozentrische ,Weltliteratur'-Begriff allerdings geöffnet, und wie bereits Goethe auch an Nordamerika dachte, besteht nicht der geringste Zweifel, dass auch die frankophonen, portugiesischund spanischsprachigen Literaturen aus Afrika oder Südamerika eingegliedert werden müssen in das, was ,Weltliteratur' heutzutage ist: ein immenses kollektives Archiv von literarischen Texten. Das bedeutet nichts anderes als eine Exterritorialisierung der europäischen Literatur, wie Vittoria Borsò mit Bezug auf die lateinamerikanische Literatur zutreffend feststellt (Borsò 2003). Zweifellos wäre hier ein enzyklopädisches Kriterium, das lediglich eine Auseinandereihung unterschiedlicher Werke zur Folge hätte, weniger wirkungsvoll als ein pragmatischer und strategischer Ansatz, der eine territoriale, sprachsoziologische, institutionelle und interkulturelle Perspektive berücksichtigt. Dieser ließe sich anpassen, um Anwendungsprogramme für den Begriff,Weltliteratur hinsichtlich des Beitrags europäischer Literaturen, ihrer klassischen Autoren und Werke zu skizzieren, die zum einen soziale und individuelle Werte vermitteln und sie kritisch diskutieren, zum anderen genuin europäische anthropologische Perspektiven beschreiben und diese durch Beispiele veranschaulichen.

\section{Kanon-Variablen}

Mit diesen Beobachtungen sind wir bereits zum Kern der aktuellen Diskussionen über den Kanon vorgedrungen, die durch eine Buchveröffentlichung von Harold Bloom (1994) einen neuen Anlauf nahmen. Gleichwohl existieren 
andere gebräuchliche Ansätze der Kanonisierung, wie Verzeichnisse mit Buchempfehlungen, Register mit grundlegenden Texten für eine Basis-Bibliothek, Best of-Listen, die sich an unterschiedliche Lesergruppen und Bildungsstände richten, insbesondere auch an solche, die in Bildungsberufen tätig sind.

Vorschläge für einen nationalen oder universalen Kanon sind reichlich vorhanden - ab und an in Kombination mit der Beschränkung auf ein literarisches Genre wie den Roman -, dazu kommen Listen mit Buchempfehlungen, mit Lieblingsbüchern von Autoren oder Redakteuren, Verlagsprojekte, Studienpläne etc. All das hat den Kanon-Begriff auf pragmatische Weise erweitert und bereichert. Im Folgenden werde ich einige Projekte von herausragender Bedeutung vorstellen.

Ein Jahrzehnt lang hat Harold Bloom in unterschiedlichen Veröffentlichungen einen Kanon erstellt - darin Quintilian nicht unähnlich, dessen zehntes Buch der Institutiones oratoriae seit dem ersten Jahrhundert unserer Zeitrechnung diese Rolle übernahm, oder auch dem Heiligen Basilius, der in einem berühmten Brief vor der Lektüre heidnischer Dichter warnte. Blooms erster Ansatz zugunsten eines universalen Kanons, dessen Dreh- und Angelpunkt die englische Literatur und die europäische literarische Tradition mit ihrem Vorzeigegenie William Shakespeare war, ist so präzise wie einschlagend. Strangeness, originality, sublimity, herausragende ästhetische Qualität und historische Bedeutsamkeit sind die grundlegenden Kriterien, die Shakespeare mit fünfundzwanzig anderen Autoren teile. Bloom setzt auf die beständigsten Werte der kollektiven Erinnerung des Abendlandes und auf ein repräsentatives Ensemble von sechsundzwanzig, starken' Autoren, deren Werke verbindlichen Charakter hätten, vor allem aufgrund ihrer universalen und produktiven Rezeption. Auch wenn diesen Ansatz einiges mit Gerald Gillespies Würdigung eines gewissen literarischen Eurozentrismus angesichts „des unaufhaltsamen kulturellen Todes des europäischen Erbes” (Gillespie 1995: 89) verbindet strittig war Blooms Weigerung, Minderheiten (z.B. in Bezug auf Ethnie, gesellschaftlichen Status oder Gender) und populäre Formate oder solche der Massenkultur anzuerkennen. Obwohl seine weite Vision der Weltliteratur sich in nachfolgenden Büchern wie How to read and why (Bloom 2000) und Genius: die hundert bedeutendsten Autoren der Weltliteratur (Bloom 2004) niederschlug, waren die Auswirkungen von The Western Canon womöglich zunächst kontraproduktiv für das Kanon-Konzept, wenn auch sehr vorteilhaft für die Profilierung alternativer Kategorien. Die Debatten, die das Buch auslöste, waren richtungsweisend, um aus heutiger Sicht erneut zahlreiche wichtige Fragen aufzuwerfen.

Hervorzuheben sind zweitens die zahlreichen Vorschläge universitärer Kanones wie der jüngste Versuch des Kieler Germanisten Albert Meier, der 
eine Liste mit unentbehrlichen Büchern für Studierende philologischer Fächer - das heißt auch für Fremdsprachenlehrer an Gymnasien und an der Universität - erstellte (Griese, Kerscher, Meier 1994). Das Ergebnis ist ein weiter akademischer Kanon, basierend auf den Kriterien der Repräsentativität gemäß den literarischen Epochen, den Sprachen und Nationen Europas, maßgebend für die westliche literarische Tradition und ihre charakteristischen Gattungen. Dieser Liste liegen implizit folgende bereits erwähnte Kriterien zugrunde: ästhetische Originalität, ethische Relevanz, Gesellschaftskritik, symbolische Funktionen wie beispielsweise eine identitätsstiftende Wirkung im Hinblick auf den Zusammenhalt sozialer Gruppen und Nationen oder ihre Anerkennung in den Augen anderer. Es handelt sich um eine historische und akademische Auswahl, die stark konzentriert ist auf den Mainstream der europäischen Literaturgeschichte (unter Berücksichtigung der amerikanischen und lateinamerikanischen Literatur).

Interessant aufgrund der Kategorisierung der Kriterien ist der Beitrag von Ulrich Schulz-Buschhaus (1975), der zwischen ästhetischen, sozialen und für literarische Studien relevanten Kanones differenziert, ohne dabei eine Liste zu erstellen. Gleichwohl wird deutlich, dass diese Klassifizierung zu einem Kanon von Bestsellern, bezogen auf Epochen, Altersstufen oder kulturelles Niveau, oder zu einem didaktischen Kanon für die Lehre in Einrichtungen des öffentlichen Bildungswesens etc. erweitert werden könnte. In diesem Sinne kann festgestellt werden, dass der seit der Romantik in Umlauf befindliche normative Kanon poetischer Werke Platz macht für die Erstellung unterschiedlicher Register im Horizont einer eigenen Programmatik, die bestimmte Gruppen oder literarische Schulen zu verschiedenen Zeiten verbreiteten, wie beispielsweise die Liste mit 56 Empfehlungen und 59 Ablehnungen, die die Surrealisten in Umlauf brachten: „Les écrivains ,recommandés“ par les surréalistes” aus dem Jahr 1930: „Lisez - Ne lisez pas” (veröffentlicht in: Losfeld 1980).

Daneben existieren Vorschläge für einen literarischen Kanon, der sich einreiht in weiter gefasste Programme eines Allgemeinwissens; dazu gehören die Entwürfe des Hamburger Anglisten Dietrich Schwanitz (1999) oder des Altphilologen Manfred Fuhrmann (2004), der einen Kanon der bürgerlichen Kultur beschreibt. Die Vorschläge von Schwanitz und Fuhrmann sind konsensfähig und sprechen ein großes Publikum an, obwohl sie Gattungen der Populärkultur ausschließen (wie Western und Kriminalromane, erotische Romane, Gruselgeschichten, Arzt-, Heimat- und historische Unterhaltungsromane, Trucker-Romane etc.). Die Werte, die Schwanitz in den siebzehn von ihm registrierten literarischen Werken verteidigt (von zehn Autoren aus acht europäischen Ländern), sind dem traditionellen humanistischen Format verpflichtet; dazu gehört beispielsweise der Beitrag des Wissens für das Verständnis der 
Welt und ihrer Nationen, für die Kenntnis von der conditio humana sowie für die Entwicklung der europäischen Kultur. Darüber hinaus bezieht Schwanitz auch philosophische und wissenschaftliche Schriften sowie Musik in das weite geistige Rüstzeug der europäischen Kultur ein.

Ein anderer in Deutschland erfolgreicher Ansatz stammt von dem lange Zeit einflussreichsten Literaturkritiker des Landes, Marcel Reich-Ranicki; er schlug 2002 ein Verzeichnis von zwanzig Romanen vor - von Goethe bis Thomas Bernhard -, die seiner Ansicht nach den Kanon des deutschsprachigen Romans bildeten. ${ }^{4}$ Der Kritiker begründete seine deutlich subjektive und zeitgenössische Titel ausschließende Romanauswahl, indem er Genuss und Wohlgefallen der privaten Lektüre einforderte (was indes nicht mit dem von Roland Barthes verfochtenen, intellektuelleren Konzept des plaisir $d u$ texte zu verwechseln ist), und rechtfertigte die Auswahl mit der durchgängigen Lesbarkeit der Werke - ihrer leichten Verständlichkeit -, ihrem hohen literarischen Wert und ihrer im Verlauf der Zeit immer wieder erneuerten Aktualität. Es handelt sich bei diesem Kanon um Empfehlungen einer weithin bekannten Autorität in literarischen Fragen, um eine Einladung zur Lektüre, die sich an ein großes, an einer (literarischen) Allgemeinbildung interessiertes Publikum richtet, wenn auch auf Kosten einer Reduktion der Komplexität der Literaturgeschichte. Die Textsammlung, die sich gleichermaßen auch für den Gymnasialunterricht und die universitäre Lehre eignet, ist nach wie vor im Handel erhältlich.

1980 hat die in Hamburg herausgegebene Wochenzeitung DIE ZEIT ein auf den ersten Blick weniger ambitioniertes Projekt lanciert, das mir allerdings symptomatisch zu sein scheint, spiegelt es doch die breite Resonanz solcher Rankings und Empfehlungen wider. In der Zeit-Bibliothek der 100 Bücher (Raddatz 2002) wurden Texte der Erzählliteratur aller Zeiten und Länder vorgestellt, jeweils einer pro Autor, ausgewählt von einem Kritikerkomitee. Ein ähnliches Projekt startete übrigens die französische Tageszeitung Le Monde, die bekanntlich ebenfalls großen Einfluss auf die öffentliche Meinung in Europa hat. 1999 legte sie ihren Lesern zweihundert Bücher des 20. Jahrhunderts vor, zuvor ausgewählt von Journalisten, Kritikern und Buchhändlern (Les cent livres $d u$ siècle). ${ }^{5}$ Die Liste, die dann dank der Abstimmung von 17.000 Lesern zustande kam, wird angeführt von Albert Camus' L'Étranger (Der Fremde) und Marcel Prousts À la recherche du temps perdu (Aufder Suche nach der verlorenen

4 Eine Anthologie dieser Titel erschien im Insel Verlag; sie wurde um Anthologien mit Erzählungen und Novellen (170 Texte, 2003), Theaterstücken (42 von 23 Autoren, 2004), Gedichten (1370 von 272 Dichtern, 2005) und Essays (255 von 166 Autoren, 2006) ergänzt: Reich-Ranicki 2002-2006.

5 http://les100livresdusiecle.com/ 
Zeit), gefolgt von Kafkas Der Prozeß und Le Petit Prince (Der kleine Prinz) von Saint-Éxupery ebenso wie Le Lotus bleu (Der blaue Lotos) aus der Comicserie Les aventures $d u$ Tintin (Tim und Struppi, Platz 18), dem Tagebuch der Anne Frank (Platz 19), Gabriel García Márquez' Cien años de soledad (Hundert Jahre Einsamkeit, Platz 33) und J.R. Tolkiens The Lord of the Rings (Der Herr der Ringe, Platz 58). Ähnliche Ergebnisse erzielt eine norwegische Zusammenstellung mit dem Titel Die hundert besten Bücher aller Zeiten, ${ }^{6}$ und in die gleiche Richtung weist die 2004 in der Zeitschrift Lire veröffentlichte Liste (Les cent livres préférés des Français), ${ }^{7}$ die ebenfalls populäre Gattungen würdigt, angefangen bei der Bibel, gefolgt von Les Misérables und Le Petit Prince bis hin zu Alexandre Dumas, Agatha Christie, Enid Blyton, Jules Verne, Jack London oder Pearl S. Buck.

Erwähnenswert sind schließlich in Deutschland initiierte Projekte wie eine „Bibliothek der 50 großen Romane des 20. Jahrhunderts“, die die Süddeutsche Zeitung auflegte; zwischen 2004 und 2005 wurden wöchentlich jeweils ein Roman oder eine Novelle angeboten. ${ }^{8}$ Auf das Ranglistenprinzip wurde zugunsten eines Pauschalangebots verzichtet, ein Prinzip, dem im gleichen Zeitraum auch die Bildzeitung mit einer 25 Bände umfassenden, auf den Massengeschmack ausgerichteten Bibliothek folgte. ${ }^{9}$ Auch die fünfzehn „Lieblingsbücher der Deutschen“, die 2004 vom Zweiten Deutschen Fernsehen (ZDF) in Kooperation mit der Stiftung Lesen sowie mit dem Börsenverein des Deutschen Buchhandels ermittelt und die in einer Ringvorlesung im Sommersemester 2005 auch an der Kieler Universität vorgestellt wurden (Jürgensen 2006), machen die mehrschichtige literarischer Typologie weitverbreiterter Lektüren in Europa deutlich. Die Liste führen Der Herr der Ringe und Die Bibel an, sie enthält Bestseller wie Das Geisterhaus von Isabel Allende und Ken Follets Die Säulen der Erde, Noah Gordons Der Medicus, J.K. Rowlings Harry Potter und der Stein des Weisen und Paulo Coelhos Der Alchimist ebenso wie anspruchsvolle, literarhistorisch relevante Werke wie Goethes Faust I und Thomas Manns Buddenbrooks. Eindeutig dominieren die Bestseller der Unterhaltungsliteratur, während Lyrik und zeitgenössische Dramatik überhaupt keine Rolle spielen. Solche Aufstellungen sind beliebig erweiterbar, wie die „Top-50-Liste”

6 Bei näherer Betrachtung bezieht sich diese 2002 veröffentlichte Liste (Tidenes 100 viktigste bøker) allerdings eher auf das 20. Jahrhundert: http://www.bokklubben.no/ SamboWeb/side.do?dokId=547432\&rom $=$ DK

http://www.lexpress.fr/culture/livre/les-100-livres-preferes-des-francais_809482.html

http://www.dieterwunderlich.de/sz_bibliothek.htm

9 http://www.weltbild.com/presse/pressemitteilung/BILD-BESTSELLER-

BIBLIOTHEK-Grosse-Romane-grosses-Gefuehl/247/ 
zeigt, die die Stiftung Lesen wenig später nachgelegt hat. ${ }^{10}$ Sie zeigen nicht mehr als Momentaufnahmen des Lesens; sie liefern keine Indizien für einen literarischen Kanon, sondern bilden das gegenwärtige Konsumverhalten der Leser $\mathrm{ab}$ - und zwar weltweit. Signifikante Unterschiede im Hinblick auf den Lesegeschmack im Bereich der „Lieblingsbücher” lassen sich nur noch bei den Klassikern ausmachen, die es in diese Listen schaffen. Goethe und beispielsweise Thomas Mann sind eine spezifisch deutsche Besonderheit und müssten dementsprechend für andere Länder durch Werke der jeweils eigenen Nationalliteratur ersetzt werden. Zuletzt dürfte in diesem Zusammenhang die Feststellung bedenkenswert sein, dass zu ihrer Entstehungszeit außergewöhnlich originelle Werke wie die Lyrik Baudelaires und Rimbauds oder Hundert Jahre Einsamkeit von Gabriel García Márquez früher oder später in den Geschmackshorizont breiter Leserschichten aufgenommen werden. Vor diesem Hintergrund lässt sich unter den Stichworten ,Kanon' und ,Anti-Kanon' auch die bürgerliche und nationale Assimilierung der künstlerischen und literarischen Avantgarden im Laufe des 20. Jahrhunderts nachzeichnen - ihre nach und nach erfolgte Musealisierung trotz des einst bewussten Bruchs mit dem etablierten Kanon (vgl. dazu Marx 2004).

Nicht zuletzt wegen der zunehmenden Orientierung solcher Listen und Zusammenstellungen an Verkaufserfolgen lohnt es sich, auf die jüngste Initiative hinzuweisen, die dagegen die literarisch relevantesten Publikationen der letzten sechzig Jahre in den Blick nahm. Die Wochenzeitung DIE ZEIT präsentierte im Juli und August 2012 sieben Wochen lang für jedes Jahrzehnt von 1945 an maßgebliche Werke aller Gattungen - Lyrik, Drama, Roman, Essay -, die jeweils den Rang eines Klassikers der Nachkriegszeit beanspruchen können und damit kanontauglich sind. ${ }^{11}$ Das Besondere an der Begründung der Auswahl ist der Hinweis auf die Frage, was Europa heute ist und welche historischen Erfahrungen im 20. Jahrhundert für die Entwicklung der literarischen Kultur und des intellektuellen Lebens maßgeblich waren. Literaten und Intellektuelle wie Albert Camus, Thomas Bernhardt, Elsa Morante, Javier Marías, Péter Esterházy, Imre Kertész, W.G. Sebald oder Patrick Modiano verfassten ihre Werke unter dem Einfluss dieser Erfahrungen. Das Fundament ihrer Reflexionen - so Iris Radisch (2012) - ist die Niederlage von 1945, die Krise Europas im Zusammenhang mit dem Verlust der Unschuld und der damit verbundenen Blamage und Selbstentzauberung. Dass unter solchen Voraussetzungen Literatur von Weltrang entstanden ist, steht außer Frage. Aber kann

\footnotetext{
${ }_{10}$ http://www.stiftunglesen.de/leseempfehlungen.html

11 http://www.zeit.de/serie/literaturkanon/seite-1
} 
es sinnvoll sein, auf der Grundlage dieser Kriterien einen zukunftsweisenden Kanon zu erstellen? Ich meine ja.

So unterschiedlich derartige Initiativen auch sein mögen, eines ist ihnen gemeinsam: Sie zeigen, wie der akademische Kanon um Texte aus Verzeichnissen populärer Lektüren erweitert wird, die sich zwar am Massenkonsum orientieren, dabei aber weder notwendigerweise trivial sein müssen noch sich an den sogenannten, Meisterwerken' ausrichten.

\section{Perspektiven: Europäische Lektüren}

Damit gelangen wir zu einem grundlegenden Punkt in unserer Kieler KanonForschung. Die Anwendbarkeit eines solchen Konzepts wird in der Zukunft von seiner Dehnbarkeit, seinem performativen Potenzial und der Fähigkeit abhängen, die realen Lesepraktiken auf allen gesellschaftlichen und institutionellen Ebenen aufzunehmen. Ein solches Lektüre-Korpus müsste sich um die anthropologischen Achsen drehen, die für die Konstruktion Europas maßgeblich sind, um eine kritische Reflexion seines kulturellen Imaginariums, seines gesellschaftlichen Horizonts und die in dieser Tradition gewachsenen Profile der conditio humana. Eine zukünftige Aufgabe des ELiCA-Projekts im Sinne der Lektüre-Förderung auf europäischer Ebene würde darin bestehen, ein solches Text-Korpus festzulegen und Projekte für seine Verbreitung anzustoßen, orientiert an den Lehr- und Bildungseinrichtungen. Hier denke ich auch an die unter dem Dach Life Long Learning entwickelten Programme, erweitert um Maßnahmen auf kommunaler Ebene, die auch Stadtviertel einbeziehen, deren Bewohner gemeinhin als ,bildungsfern' bezeichnet werden. Mein Vorschlag wäre daher eine Dezentralisierung des Kanons oder besser gesagt: eine Modularisierung, angepasst an die jeweiligen kulturellen Horizonte der Zielgruppen dieses Lektüreangebots.

Die Anregung, das Kanon-Konzept zugunsten einer Kategorie ,LektüreKorpus europäischer Literatur' zu erweitern, setzt die produktive Auseinandersetzung mit Kategorien wie ,Klassiker', und ,Weltliteratur' voraus, deren Fortführung auch die Konkretisierung oder Materialisierung in anthologischen Projekten sein könnte. Diese dürften im Digitalzeitalter umso sinnvoller erscheinen, da das in Büchern überlieferte, von Kindheit oder Jugend an durch Lektüre angeeignete kulturelle Kapitel zunehmend an Bedeutung verliert.

Zwei Untersuchungen im verwandten Umfeld unseres Engagements für einen literarischen Kanon Europas wären dabei als Orientierungshilfe dienlich. Einerseits das Lektüremodell, das Harold Bloom im Vorwort von How to read and why? (2000) skizziert: Lesen verstanden als einsame, stille und tiefsinnige 
Übung, emotional und existenziell zugleich, und durchgeführt nicht ohne eine gewisse Dosis (Selbst)Ironie. Dabei fasst Bloom Werke ins Auge, die den Horizont des Lesers, seine Alltagswirklichkeit und seine Selbstwahrnehmung erweitern und deren Figuren ein Panorama möglicher Identifikation(en) öffnen - über die bloße Unterhaltung hinaus. Auf der anderen Seite wäre Italo Calvino heranzuziehen, der das Lesen vielleicht am besten charakterisiert hat als Grenzerfahrung und Initiationserlebnis an den Schnittstellen, an denen klassische Autoren, Kanon und ,Weltliteratur' mit der individuellen Erfahrung des Lesers in Kontakt treten (Calvino 2003). Calvino lehnt Kategorisierungen $\mathrm{ab}$, die das Klassische als ästhetische Meisterleistungen definieren (im Sinne außergewöhnlicher Einheit und modellhafter Harmonie, wie Fuhrmann 2004 festlegte (2004: 40-42)), und hält dem eine individuelle Praxis der Lektüre entgegen, indem er fünfzehn Argumente zugunsten von Büchern anführt, die im kollektiven Gedächtnis und im individuellen Unterbewusstsein nachhaltig präsent sind und gleichermaßen zur individuellen wie kollektiven Selbstbestimmung beitragen. Sie legen einen Lektürefundus fest, der auf Dauer aktuell bleibt - aufgrund seiner sich ständig erneuernden Gültigkeit, seiner kontinuierlichen Wirkungskraft im Laufe der Jahrhunderte oder auch in der individuellen Lebensgeschichte des Einzelnen. Auf diese Weise unterbreitet Calvino einen so intelligenten wie flexiblen Vorschlag, der die Fallen der Macht im politischen und gesellschaftlichen Diskurs ${ }^{12}$ zu umgehen weiß, indem er geschickt einer eurozentrischen Perspektive ausweicht.

Ein so verstandener Kanon öffnet literarischen Texten die Tür, die auch Minderheiten oder marginalisierten Gruppen in bestimmten kulturellen $\mathrm{Zu}$ sammenhängen einen identifikatorischen Rahmen bereitstellen (zum Beispiel aus genderspezifischen und sprachsoziologischen Gründen sowie durch die Berücksichtigung ethnischer, territorialer oder allgemein gesellschaftlicher Kontexte). Zugleich kann ein solcher Kanon als literarisches Referenzsystem für heute randständige, historisch minorisierte Kulturen dienen. Dies gilt zum Beispiel für die in Europa mit der europäischen Romantik (wieder-)auflebenden Kulturen mit entsprechenden literarischen Diskursen, wie es in Estland

12 Gerade in Zusammenhang mit Lateinamerika ist nicht zu übersehen, dass sich literarische Kanonbildung allzu oft den Vorgaben der Macht unterworfen hat, wie die Beiträge eines von Christian Wetzlaff-Eggebert und Martin Traine herausgegebenen Bandes feststellen (Wentzlaff-Eggebert, Traine 2000). Umso sinnvoller erscheint vom kulturwissenschaftlichen Standpunkt aus Tania Franco Carvalhals Plädoyer für eine dialektische Dynamik eigener und fremder Kräfte bei den iberoromanischen Literaturen; um sie zu erfassen, müssen die gleichzeitig wirkenden Dimensionen des Lokalen, Regionalen und Globalen berücksichtigt werden (Franco Carvalhal 1995). 
oder Galicien der Fall ist, deren Entwicklung seither vergleichbar ist und die im Moment ihrer modernen Gründung und kulturellen Fundierung im Zusammenhang mit Nationsbildungsprozessen (in beiden Fällen angestoßen von Dichterinnen wie Lydia Koidula und Rosalía de Castro in der zweiten Hälfte des 19. Jahrhunderts) beachtliche Bedeutung gewonnen haben. Ebenso wenig dürfte die Relevanz und Wirkungskraft eines solchen Lektüre-Korpus für einzelne Generationsgruppen oder für großstädtische und vorstädtische tribes (oft jugendliche Szenegruppen im Sinne der culturas híbridas nach Nestor García Canclini $(1990,2007)$ ) vergessen werden. Der Vorschlag von Calvino lässt auch genug Spielraum, Migranten und ihre jeweiligen kulturellen Imaginarien in ein Lektüre-Korpus einzubeziehen, und bemüht sich so implizit um die Herstellung eines Gleichgewichts zwischen den Kontinenten. Ohne Zweifel erlaubt Calvinos Leitfaden der fünfzehn Thesen darauf zu verweisen, dass jede Beziehung zwischen Zentrum und Peripherie in Kultursystemen als ein sich ständig wandelndes, relationales Verhältnis angesehen werden muss, dessen symbolische Dimension Gegenstand steter Verhandlung durch die unterschiedlichen sozialen und ethnischen Gruppen ist. Zu guter Letzt lässt dieses Konzept eines variablen Lektüre-Korpus der immensen Bedeutung von Sprache und Literatur für das Überleben von Völkern oder sozialen Minderheiten Gerechtigkeit widerfahren, denn Sprache und Literatur sind bekanntlich Übermittler der eigenen kulturellen Register, mentaler Bilder und identitätsstiftender Konzepte. Deshalb sind sie umso bedeutender in subalternen Kultursystemen, die in einer kontinuierlichen und wechselhaften Verhandlung mit den hegemonischen Instanzen der Macht und mit anderen rivalisierenden kulturellen Diskursen stehen (wie beispielsweise in einigen der autonomen Regionen Spaniens (Cabo 2012) oder in Estland, ${ }^{13}$ die hier stellvertretend für kleine Nationalliteraturen stehen ${ }^{14}$ ).

Calvinos fünfzehn Thesen können daher auch als Richtlinie dienen, um das Ungleichgewicht zwischen institutionalisierten Lesegewohnheiten an Gymnasium (oder an anderen Schulen) und an der Universität einerseits und der privaten Lektüre andererseits zu beheben, indem sie die Kluft schließen, die sich zwischen gebildeter und trivialer Lektüre, zwischen Bestsellern und

13 Der Band Interlitteraria 17 (University of Tartu Press, 2012) versammelt mehrere Beiträge zum Zentrum-Peripherie-Verhältnis sowie zum Standort der estnischen Literatur im Kontext der Weltliteratur, neben anderen Heero 2012, Lukas 2012, Merilai 2012, Talvet 2012.

14 So stellt Gerald Gillespie (1995: 91) diesbezüglich eine Verknüpfung zwischen großstädtischen und „regionalen” literarischen Subkulturen her, und zwar aufgrund ihrer diskursiven Subalternität gegenüber den Leitkulturen. 
GÓMEZ-MONTERO

literarischer Avantgarde auftut, wie die ELiCA-Umfragen gezeigt und wie wir es auf der Projekt-Homepage www.uni-kiel.de/elica dokumentiert haben.

\section{Conclusio: Offene Fragen}

Natürlich warten einige relevante Fragen noch auf konsensfähige Antworten. Was genau verstehen wir unter einem literarischen Europa? Wie definiert sich eine europäische Identität, die in der Literatur reflektiert wird? Grundlegende Untersuchungen dazu wurden bereits im Rahmen einer vom römischen ELiCAPartner geleiteten Tagung durchgeführt, die dem Projekt University and School for a European Literary Canon und dessen Symposion „La letteratura e la formazione degli europei" ${ }^{15}$ vorausging; die Ergebnisse dieser ersten Tagung hat Roberto Antonelli (2008) in dem informativen Band Il canone europeo zusammengefasst. Hilfreich in dieser Hinsicht sind auch Bände wie Cercando l'Europa (Paradisi, Punzi, Tomassetti 2010) und Identité littéraire de l'Europe, ${ }^{16}$ in denen Fragen aufgeworfen und Perspektiven eröffnet werden, die sich mit Überlegungen zur Funktion der Kultur als Fundament Europas - seiner Geschichte und seiner Gesellschaft(en) - verknüpfen lassen, erweitert durch die Schriften europäischer Intellektueller wie Rémi Brague (Brague 2009), Edgar Morin (Morin 1987), Jorge Semprún (Semprún 2006), Manfred Fuhrmann (Fuhrmann 2002), Jürgen Habermas (Habermas 2011) oder Hans Magnus Enzensberger (Enzensberger 2007), die sich allesamt mit der kulturellen Identität Europas beschäftigen. Der Anfang dieser Reihe von Europa-Denkern des ausgehenden 20. Jahrhunderts muss weniger in der Romantik als vielmehr in jener Denktradition aufgesucht werden, die im Scheitern Europas verwurzelt ist. Als Gründungsvater solcher kulturanthropologischen Entwürfe kann wohl Paul Valéry zitiert werden, der 1922 in einem Vortrag auf die „signification fonctionnelle" Europas und des Europäischen hingewiesen hat, und zwar über das Territorium und die Geschichte Europas hinaus. Der Vortrag wurde erst 1924 unter dem Titel „Caractères de l'esprit européen” (1934 ein weiteres Mal als „L'Européen“) publiziert. ${ }^{17}$ Valéry stellt hier fest, dass die römische Gesetzgebung, „la moral subjective” des Christentums und die „méthode de penser” der Griechen den umtriebigen Homo europæus mit seiner „inquiétude

15 Die internationale Konferenz „La letteratura e la formazione degli europei” fand vom 16. bis 17. März 2012 an der Sapienza Università di Roma statt. Siehe dazu: Antonelli, Materni, Paradisi 2012.

16 Fumaroli 2000, mit Beiträgen von Yves Bonnefoy, Maria Corti und Karlheinz Stierle et al.

17 Paul Valéry, „La crise de l'esprit” (Valéry 1957: 988-1000), „Note (ou l’Européen)” (ib. 1000-1014) ; vgl. auch „La politique de l'esprit” (ib. 1014-1040). 
et recherche perpetuelles" ${ }^{18}$ auszeichnen. Seine Zeit, so Valéry, erlebe indes die Agonie Europas, ${ }^{19}$ weshalb in den Begriff der europäischen Literatur - dies als weiterführende Konsequenz - auch die Schattenseiten ihrer Geschichte eingeschrieben werden müssen: die Kolonialpolitik, eine problematische Dekolonisierung und insbesondere die Verwandlung des gestalterischen Potenzials in das Zerstörungswerk der Kriege im 20. Jahrhundert.

Wenn wir unsere Ansätze und Schlussfolgerungen in den Rahmen solcher Europa umspannenden Untersuchungen stellen wollen, müssen wir einerseits die literarischen Kulturen Ost- und Südosteuropas stärker in diesen Horizont mit einbeziehen, anderseits aber auch die Peripherien großstädtischer Ballungsräume, die als kulturell hybride Territorien gelten, berücksichtigen, wenn es um den Begriff einer ,europäischen Kultur' geht. Diese Räume sind nicht weniger kreativ und dynamisch, und hier wiederholt sich ein Prozess, den Edgar Morin als dialogisches Zusammenwirken konträrer Kräfte beschrieben hat, welches für das historischen Werden Europas charakteristisch sei: „C'est la dialogique qui est au cœur de l'identité culturelle européenne.” (Morin 1987: 150) In historischer Perspektive gehören dazu Religion und Vernunft, mythisches und kritisches Denken, das Individuelle und Persönliche gegenüber dem Sozialen und Universellen. Gleichzeitig verpflichtet die neue Situation der Multi- und Transkulturalität Europa dazu, sich der Herausforderung seines selbstkritischen Vermögens und seines Potenzials zur ständigen Neuerfindung zu stellen. Beides hat Ulrich Beck (Beck, Grande 2004) neben dem Bewusstsein, unterschiedlichen Bezugssystemen anzugehören und identitätsstiftende Horizonte koordinieren zu müssen, als Merkmale Europas herausgestellt, ohne die daraus folgenden Konflikte zu vergessen, die noch zu lösen sind.

Dazu können die literarischen Diskurse in Europa einen wesentlichen Beitrag leisten, und unser Vermittlungsprojekt zwischen dem Oberstufenunterricht und der Literaturvermittlung an Universitäten hat es sich zur Aufgabe gemacht, Instrumente und Grundlagen beizusteuern, um die Rolle der Literatur in Europa ins Bewusstsein zu rücken: als Ort der Verhandlung von Wissen, Selbsterkenntnis und ethischem Bewusstsein, der Veranschaulichung gesellschaftlicher und historischer Entwicklungen ebenso wie der gegenwärtigen interkulturellen Bereicherung, um nur einige wenige Stichworte zu nennen. Gegen den Standpunkt und die Zugangsweise des EliCa-Projekts ließe sich gewiss einwenden, dass sich in den vorgestellten Ergebnissen ein romanischer

18 Paul Valéry, „La crise de l'esprit” (ib. 996).

19 Paul Valéry, „La crise de l'esprit” (ib. 990); diesen Gedanken greift übrigens die spanische Denkerin Maria Zambrano auf; ihre Essays erschienen zwischen 1940-44 (Zambrano 2000, 2004). 
Vitalismus bemerkbar macht, dessen Verwurzelung in der am Mittelmeer entstandenen griechisch-römischen Zivilisation offensichtlich ist. Diese Zivilisation hat bis heute Bildungsmodelle hervorgebracht, die vom Vertrauen in den Menschen getragen sind. Es liegt nahe, den vorliegenden Entwurf mit solchen Menschenbildern und Geschichtsmodellen abzugleichen, die eher von einem melancholischen Humanismus bestimmt sind und eine Kulturanthropologie vertreten, die einer via negativa des modernen Denkens und somit einer radikaleren Kritik am Menschen, an der Geschichte und an der Gesellschaft verpflichtet ist, an der auch die Literatur seit Beginn der Moderne partizipiert.

Indes bin ich zugleich sicher, dass wir den institutionellen Rahmen, in dem wir uns bewegen, nutzen sollten; er ermöglicht - trotz aller Unzulänglichkeiten - performative Impulse für die Festlegung eines humanistischen Kanons, das heißt eines Referenzsystems, das komplexere Bilder des Menschen beisteuern kann als jene Texte, die tagtäglich von der Konsumgesellschaft und den Massenmedien verbreitet werden. Sich selbst und den anderen kennenzulernen, das Verständnis für einander zu vertiefen, auch das könnte die Aufgabe eines literarischen Kanons für Europa sein.

\author{
Javier Gómez-Montero \\ gomez-montero@romanistik.uni-kiel.de \\ Christian-Albrechts-Universität zu Kiel \\ Romanisches Seminar \\ Leibnizstr. 10 \\ D-24098 Kiel \\ DEUTSCHLAND
}

\title{
Bibliographie
}

Antonelli, R. 2008. La letteratura europea, ieri, oggi, domani. - S. Bianchini, A. Landolfi, Hg., Il canone europeo, Rom: Viella, 9-40.

Antonelli, R., Materni, M., Paradisi, G., Hg. 2012. La letteratura e la formazione degli europei. Roma: Bagatto Libri.

Arnold, H. L., Hg. 2002. Literarische Kanonbildung, München: Ed. Text + Kritik.

Bachmann-Medick, D. 2004. Multikultur oder kulturelle Differenzen? Neue Konzepte von Weltliteratur und Übersetzung in postkolonialer Perspektive. - D. BachmannMedick, Hg., Kultur als Text. Die anthropologische Wende in der Literaturwissenschaft. Tübingen/Basel: Francke, 262-296.

Baratta, F., Grossi, L., Pupolin, L., Hg. 2012. Dossier quadrimestrale del centro di iniziativa democratica degli insegnanti. I giovani europei e la letteratura - indagine, percorsi, canone. Rom: ciid.

Barthes, R. 1973. Le plaisir du texte. Paris: Seuil. 
Europäische Lektüren jenseits von Europa

Beck, U., Grande, E. 2004. Das kosmopolitische Europa. Gesellschaft und Politik in der Zweiten Moderne. Frankfurt am Main: Suhrkamp.

Berger, G., Lüsebrink, H.-J., Hg. 1987. Literarische Kanonbildung in der Romania. Rheinfelden: Schäuble. [Darin: „Kanonbildung in systematischer Sicht“, 3-32.]

Birus, H. 1995. Goethes Idee der Weltliteratur. Eine historische Vergegenwärtigung. - M. Schmeling, Hg., Weltliteratur heute. Konzepte und Perspektiven. Würzburg: Königshausen \& Neumann, 5-28.

Bloom, H. 1994. The Western Canon: The Books and School of the Ages. New York: Harcourt Brace.

Bloom, H. 2000. How to Read and Why. New York: Scribner.

Bloom, H. 2004. Genius: die hundert bedeutendsten Autoren der Weltliteratur. München: Knaus.

Bogdal, K.-M., Kammler, C., Hg. 2000. (K)ein Kanon. 30 Schulklassiker neu gelesen. München: Oldenbourg.

Bohnenkamp, A. 1999. Den Wechseltausch zu befördern. Goethes Entwurf einer Weltliteratur. - A. Bohnenkamp, Hg., Goethe. Ästhetische Schriften 1824-1832: Über Kunst und Altertum V-VI, Bd. 22. Frankfurt am Main: Dt. Klassiker-Verlag, 937-964.

Borsò, V. 2003. Europäische Literaturen versus Weltliteratur - Zur Zukunft der Nationalliteratur. - Jahrbuch der Heinrich-Heine-Universität Düsseldorf 2003, http://www.uni-duesseldorf.de/home/Jahrbuch/2003/Borso.

Boxall, P. 2007. 1001 Bücher, die Sie lesen sollten, bevor das Leben vorbei ist. Zürich: Olms.

Brague, R. 2009. Europe, la voie romaine. Paris: Gallimard.

Cabo Aseguinolaza, F. 2012. El lugar de la literatura española. Barcelona: Crítica.

Calvino, I. 2003. Warum Klassiker lesen? [Original „Perché leggere i classici?” (L'espresso, 28.6.1981).] - Warum Klassiker lesen? München: Hanser, 7-14.

Damrosch, D., Hg. 2009. Teaching World Literature. New York: Modern Language Assoc. of America.

D’haen, T., Goerlandt, I., Hg. 2009. Literature for Europe? Amsterdam: Rodopi.

Enzensberger, H. M. 2007. Ach, Europa! Wahrnehmungen aus sieben Ländern. Mit einem Epilog aus dem Jahre 2006. Frankfurt am Main: Suhrkamp.

Franco Carvalhal, T. 1995. L'universel, le national et le régional dans la littérature brésilienne. - M. Schmeling, Hg., Weltliteratur heute. Konzepte und Perspektiven. Würzburg: Königshausen \& Neumann, 49-58.

Fuhrmann, M. 2002. Bildung. Europas kulturelle Identität. Stuttgart: Reclam.

Fuhrmann, M. 2004. Der europäische Bildungskanon. Frankfurt am Main: Insel.

Fumaroli, M. 2000. Identité littéraire de l'Europe. Paris: PUF.

García Canclini, N. 1990. Culturas híbridas: estrategias para entrar y salir de la modernidad, Mexico: Grijalbo.

García Canclini, N. 2007. Imaginarios urbanos. Buenos Aires: Eudeba.

Gillespie, G. 1995. Auf den multikulturellen Irrwegen der amerikanischen Komparatistik: Kontrast und Mahnbild für ein junges Europa. - M. Schmeling, 
GÓMEZ-MONTERO

Hg., Weltliteratur heute. Konzepte und Perspektiven. Würzburg: Königshausen \& Neumann, 85-100.

Gómez-Montero, J. 2012. Lecturas europeas mas allá de Europa: Weltliteratur y canon literario. - R. Antonelli, M. Materni, G. Paradisi, Hg., La letteratura e la formazione degli europei. Roma: Bagatto Libri, 33-47.

Griese, S., Kerscher, H., Meier, A., Hg., 1994. Die Leseliste. Kommentierte Empfehlungen. Stuttgart: Reclam.

Habermas, J. 2011. Zur Verfassung Europas: ein Essay. Berlin: Suhrkamp.

Heero, A. 2012. Timotheus Polus and Reiner Brockmann: the Nexus Between the Estonian and European Literary Discourse. - Interlitteraria, 17, 366-376.

Hesse, H. 1987. Gesammelte Werke in zwölf Bänden, Bd. 11: Eine Bibliothek der Weltliteratur. Frankfurt am Main: Suhrkamp.

Heydebrand, R. v., Hg. 1998. Kanon-Macht-Kultur. Theoretische, historische und soziale Aspekte ästhetischer Kanonbildungen. Stuttgart: Metzler.

Jürgensen, Ch., Hg. 2006. Die Lieblingsbücher der Deutschen. Kiel: Ludwig.

Koch, M. 2002. Weimaraner Weltbewohner. Zur Genese von Goethes Begriff Weltliteratur. Tübingen: Niemeyer.

Kochan, D. C., Hg. 1990. Literaturdidaktik-Lektürekanon-Literaturunterricht. Amsterdam/ Atlanta: Rodopi.

Lamping, D. 2010. Die Idee der Weltliteratur. Ein Konzept Goethes und seine Karriere. Stuttgart: Kröner.

Lamping, D., Zipfel, F. 2005. Was sollen Komparatisten lesen? Berlin: Schmidt.

Lermen, B., Hg. 2003. Literatur, Werte und europäische Identität: Brücke zu einem vereinten Europa; Dokumentation der internationalen Fachtagung der Konrad-Adenauer-Stiftung und der Karls-Universität Prag, 28.11.-1.12.2002 in Prag. Prag: Konrad-AdenauerStiftung.

Losfeld, É., Hg. 1980. Tracts surréalistes et déclarations collectives (1922-1969), 1 (19221939). Paris: Le terrain vague.

Lukas, L. 2012. The Shifting Position of German Literature in the Estonian Canon of World Literature. -Interlitteraria, 17, 225-240.

Manger, K., Hg. 2003. Goethe und die Weltkultur. Heidelberg: Winter.

Matthes, L. 1987. Literatur im Französischunterricht. Zum Autorenkanon schulischer Curricula in der Bundesrepublik (1945-1984). - G. Berger, H.-J. Lüsebrink, Hg., Literarische Kanonbildung in der Romania. Rheinfelden: Schäuble, 247-272.

Marx, W. 2004. Les arrière-gardes au XX ${ }^{\text {ème }}$ siècle. Paris: PUF.

Mattéi, J.-F. 2011. Le procès de l'Europe: grandeur et misère de la culture européenne. Paris: Presses universitaires de France.

Merilai, A. 2012. Estonian Poetic Surrealism: Laaban and Ehin. - Interlitteraria, 17, 167179.

Moog-Grünewald, M., Hg. 1997. Kanon und Theorie. Heidelberg: Winter.

Moretti, F. 1993. La letteratura europea. Torino: Einaudi.

Morin, E. 1987. Penser l'Europe. Paris: Gallimard.

Neuhaus, S. 2002. Revision des literarischen Kanons. Göttingen: Vandenhoeck \& Ruprecht. Paradisi, G., Punzi, A., Tomassetti, I., Hg. 2010. Cercando l'Europa. Volume temático di "Critica del testo". Roma: Viella. 
Europäische Lektüren jenseits von Europa

Poltermann, A., Hg. 1995. Literaturkanon-Medienereignis-kultureller Text. Formen interkultureller Kommunikation und Übersetzung. Berlin: Schmidt.

Pozuelo Ivancos, J. M. 2000. Teoría del canon y literatura española. Madrid: Cátedra.

Raddatz, F. J., Hg. 2002. Die Zeit - Bibliothek der 100 Bücher. Frankfurt am Main: Suhrkamp.

Radisch, I. 2012. Europas Weltliteratur. - Die ZEIT, 12.07.

Reich-Ranicki, M., Hg. 2002-2006. Der Kanon: die Deutsche Literatur. Frankfurt am Main: Insel.

Riesz,J., Hg. 1986. Literarische Kanonbildung. Canonisation littéraire. Literary Canonisation. Bayreuth: Ellwanger.

Rüdiger, H. 1990. Goethe und Europa. Essays und Aufsätze 1944-1983. Berlin: de Gruyter.

Schmeling, M. 1995. Ist Weltliteratur wünschenswert? Fortschritt und Stillstand im modernen Kulturbewußtsein. - M. Schmeling, Hg., Weltliteratur heute. Konzepte und Perspektiven. Würzburg: Königshausen \& Neumann, 153-178.

Scholz, B. F. 1987. Literarischer Kanon und literarisches System. - G. Berger, H.-J. Lüsebrink, Hg., Literarische Kanonbildung in der Romania. Rheinfelden: Schäuble, 55-86.

Schreiber, N. 2011. Europa weiter erzählen. Klagenfurt: Wieser.

Schulz-Buschhaus, U. 1975. Der Kanon der romanistischen Literaturwissenschaft. Wissenschaftsgeschichtliche Bemerkungen zum Wandel von Interessen und Methoden. Trier: NCO.

Schwanitz, D. 1999. Bildung. Alles, was man wissen muß. Frankfurt am Main: Eichborn.

Semprún, J. 2006. Pensar en Europa. Barcelona: Tusquets.

Sommerfeldt, K., Hg. 2011. Spanische Methodik. Handbuch für die Sekundarstufe I und II. Berlin: Cornelsen Verlag.

Steinmetz, H. 1985. Weltliteratur - Umriß eines literaturgeschichtlichen Konzepts. Arcadia, 20, 2-19.

Stenzel, H. 1987. Molière nach 300 Jahren? Anmerkungen zu Genese und Funktion einer Kanonisierung als Klassiker. - G. Berger, H.-J. Lüsebrink, Hg., Literarische Kanonbildung in der Romania. Rheinfelden: Schäuble, 127-144.

Strich, F. 1957. Goethe und die Weltliteratur. Bern: Francke.

Struger, J., Hg. 2008. Der Kanon - Perspektiven, Erweiterungen und Revisionen. Wien: Praesens.

Stuck, E. 2004. Kanon und Literaturstudium. Theoretische, historische und empirische Untersuchungen zum akademischen Umgang mit Lektüre-Empfehlungen. Paderborn: Mentis.

Talvet, J. The Lingering Journey of Poetry from 'Peripheries' to 'Centres': the Estonian Case of F. R. Kreutzwald’s Epic Kalevipoeg (1861) and Juhan Liiv’s (1864-1913) Lyrical Work. - Interlitteraria, 17, 93-107.

Valéry, P. 1957. La crise de l'esprit ; Note (ou l'Européen). - P. Valéry, Euvres I. Paris: Éditions Gallimard, 988-1014.

Wentzlaff-Eggebert, Ch. 1998. Eurolit. Les études littéraires en Europe. Actes du Congrès de Cologne 14-15 juin 1996. Köln: Universität zu Köln.

Wentzlaff-Eggebert, Ch., Traine, M, Hg. 2000. Kanon und Macht in Lateinamerika. Köln: Arbeitskreis Spanien-Portugal-Lateinamerika. 
GÓMEZ-MONTERO

Zambrano, M. 2000. La agonía de Europa [3ª edición]. Madrid: Trotta.

Zambrano, M. 2004. Der Verfall Europas [hrsg. und aus dem Spanischen übersetzt von Charlotte Frei]. Wien: Turia \& Kant.

Zschirnt, Ch. 2004. Bücher. Alles, was man lesen muss. München: Heyne.

\section{Wörterbücher und Internetquellen}

Arnold, H. L. 2009. Kindlers Literatur-Lexikon. Stuttgart: Metzler.

Didier, B. 1994. Dictionnaire universel des littératures. Paris: PUF.

Frenzel, E. 2005. Stoffe der Weltliteratur: ein Lexikon dichtungsgeschichtlicher Längsschnitte. Stuttgart: Kröner.

Pongs, H. 1984. Lexikon der Weltliteratur: Handwörterbuch der Litertur von A - Z. Wiesbaden: Englisch.

Wilpert, G. v., Hg. 1993. Lexikon der Weltliteratur, Bd. 2: Hauptwerke der Weltliteratur in Charakteristiken und Kurzinterpretationen. Stuttgart: Kröner.

Delaroche, Ph. 2004. Les cent livres préférés des Français. - Lire, 01.10, http://www.lexpress.fr/culture/livre/les-100-livres-preferes-des-francais_809482.html (11.03.13)

Les cent livres du siècle [Le Monde, 1999], http://les100livresdusiecle.com/ (11.03.13)

http://www.bokklubben.no/SamboWeb/side.do?dokId=547432\&rom=DK

(11.03.13)

http://www.zeit.de/serie/literaturkanon/seite-1 (26.02.13)

http://www.weltbild.com/presse/pressemitteilung/BILD-BESTSELLER-

BIBLIOTHEK-Grosse-Romane-grosses-Gefuehl/247/ (26.02.13)

http://www.dieterwunderlich.de/sz_bibliothek.htm (26.02.13)

http://www.stiftunglesen.de/leseempfehlungen.html (26.02.13)

http://www.uni-kiel.de/elica (10.07.12)

http://de.wikipedia.org/wiki/Der_Kanon (20.03.12)

http://www.derkanon.de/ (07.03.12)

http://fr.wikipedia.org/wiki/Les_100_livres_du_siècle (07.03.12)

http://lolalit.canalblog.com/archives/2011/06/19946818.html (07.03.12) 